SIR: I am puzzled by Dr Ryle's letter. I have tended to follow the widely-held view that the cutting-off experience of depersonalisation was a mental mechanism for dealing with intolerable emotions, particularly depression, anger, and anxiety. In Case 1 of Dr Ryle's letter it seems hard to believe that the young man's feelings at the railway station before he experienced depersonalisation were entirely tranquil; in Case 2, Dr Ryle describes anger as preceding depersonalisation. It seems perfectly possible, therefore, that these patients might have been hyperventilating before the depersonalisation developed. Once it develops, it may engender further anxiety, so that there is often a vicious circle.

My criterion for diagnosing hyperventilation is that the patient was hyperventilating in front of me or gave a convincing account including a demonstration of what had happened previously. This would usually be supported by other well-known collateral symptoms of hyperventilation such as a tingling in the fingers or tightness in the chest. With regard to the effect of a brown paper bag on depersonalisation, I would like to say in the first place that I have never used a brown paper bag nor do I think it is ever necessary to use one, although it is so often quoted as being 'traditional'; what you usually need to do is to calm the patient down. Hyperventilation is one among many symptoms of anxiety and it is the anxious patient who needs treatment, not the symptom. However, Dr Ryle's question is of course the key one, and we are trying to find out whether variation in the level of $\mathrm{pCO}_{2}$ in a controlled way can have an influence on this symptom. This is a part of the study to which my letter referred.

London Hospital Medical College

SAmuel I. Cohen

Turner Street

London E1 2AD

\section{Age disorientation in chronic schizophrenia}

SIR: In their recent article on age disorientation in chronic schizophrenic patients, Buhrich et al (Journal, April 1988, 152, 466-469) report that this cognitive impairment is not due to past physical treatments, or mental retardation before the onset of their illness. They consider it unlikely that an Alzheimer-type dementia may have developed independently in these patients. We may add further information by reporting a follow-up study we undertook to assess the prognostic significance of age disorientation.

Thirty older chronic schizophrenic in-patients with age disorientation were identified on the longstay units of Veterans Administration Medical
Center at Northport, New York. They were compared with 29 chronic schizophrenic patients without age disorientation and 23 patients suffering from early stages of organic dementia of known aetiology (Alzheimer's disease and multi-infarct dementia for the most). The three groups, comparable in age (mean age 60 years), were followed up in the same environment for a period of 5 years. Schizophrenic patients received standard neuroleptic medication, and all participated in daily reality orientation therapy. Weekly clinical evaluations were made by psychiatrists, and semi-annually the Clifton Geriatric Schedule and the Shortened Stockton Geriatric Rating Scale were administered by independent raters (Pattie \& Gilleard, 1978).

The results showed that at the beginning of the study, for each variable the age-disorientated schizophrenics and the demented patients were more impaired than the not-age-disorientated schizophrenics $(P<0.01)$. Five years later, all three groups were significantly different $(P<0.02)$. Schizophrenic patients without age disorientation were the least impaired and the demented patients the most impaired, with the age disorientated schizophrenic patients in-between. Analysis over time indicated that both schizophrenic groups remained stable on the cognitive scales despite fluctuations in other typical schizophrenic symptoms, and only the demented patients continued to deteriorate $(P<0.01)$.

It was concluded that two distinct cognitive levels are characteristic of chronic schizophrenia. Older schizophrenic patients given medication and daily reality-orientation therapy neither improve nor deteriorate on cognitive functions preserved. It is suggested that in schizophrenia the active organic brain disease subsides at an earlier age, probably during the onset period. The resulting brain damage in some, as shown in cognitive defects, becomes irreversible, but contrary to Alzheimer-type dementias not progressive.

A preliminary report of this study was presented at the Eighth World Congress of Psychiatry, Vienna, Austria, in August 1983.

Veterans Administration Medical Center

Paul E. Yarden

Charles S. RaPS

Northport

New York, 11768

USA

\section{Reference}

Pattie, A. H. \& Gilleard, C. J. (1978) The two year predictive validity of the Clifton assessment schedule and the shortened Stockton geriatric rating scale. British Journal of Psychiatry. 133, 457-460. 